\section{Obesidad y sobrepeso infantil}

Obesity and child overweight

Artículo resultado de proyecto de investigación financiado por

Ia Universitaria de Guayaquil

Michelle Nathaly Arzube Almeida

Univesidad de Guayaquil

https://orcid.org/0000-0002-2090-

3763

michelle.arzubea@ug.edu.ec

Guayaquil - Ecuador

Gabriela Belén Espinosa Arreaga

Univesidad de Guayaquil

https://orcid.org/0000-0001-9360-

9015

gabriela.espinosaa@ug.edu.ec

Guayaquil - Ecuador

http://www.jah-

journal.com/index.php/jah

Journal of American health, Januaty

June vol. 1. Num. 1 - 2018

Esta obra está bajo una Licencia

Creative Commons

Atribución-NoComercial-

CompartirIgual 4.0 Internacional.

RECIBIDO: 25 DE OCTUBRE 2017

ACEPTADO: 1 DE DICIEMBRE 2017

PUBLICADO: 4 DE ENERO 2018

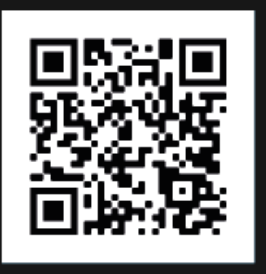

Scan this QR

code with your

smart phone or

mobile device to

read more papers

\section{RESUMEN}

El presente documento, "Sobrepeso y obesidad infantil. Es para orientar, prevenir, para que vean tratamiento en Atención a la Salud", en la nutrición adecuada para los niños en la Infancia como apoyo para la atención de niños con sobrepeso $u$ obesidad, así como para orientación de acciones comunitarias de promoción de la salud como estrategia de advertencia del sobrepeso y la obesidad. La obesidad y el sobrepeso infantil es una gran problemática internacional que ha crecido en las postreras décadas. Unas de las causas que ocasiona son los inapropiados usos de alimentarios y el sedentarismo. La finalidad común de esta investigación es saber cuáles son las causas de la obesidad y sobrepeso infantil y buscar propuestas educativas para disminuir la obesidad infantil.

PALABRAS CLAVE: Obesidad infantil, Sobrepeso, factores de riesgo, sedentarismo, mala alimentación.

\section{ABSTRACT}

This document, "Overweight and childhood obesity. It is to guide, prevent, to see treatment in Health Care", in adequate nutrition for children in childhood as support for the care of overweight or obese children, as well as for guidance of community actions to promote health as a warning strategy of overweight and obesity. Obesity and childhood overweight is a major international problem that has grown in the last decades. Some of the causes it causes are inappropriate uses of food and sedentary lifestyle. The common purpose of this research is to know what the causes of childhood obesity are and overweight and look for educational proposals to reduce childhood obesity. 


\author{
KEY WORDS Childhood obesity, \\ Overweight, risk factors, sedentary \\ lifestyle, poor diet.
}

\title{
INTRODUCCIÓN
}

La salud y la nutrición de las madres en su ciclo de embarazo, a lo largo de éste y la nutrición del niño(a) en los primordiales meses son vital para la prevención de enfermedades no traspasable. La lactancia natural es única durante los primeros 6 meses de vida la alimentación es suplementario idóneo en nutrientes y ayuda calórico contribuyente para el crecimiento físico y mental. Entre las enfermedades crónicas no transmisibles merece obtener una especial atención la obesidad infantil.

La obesidad es consecuencia de la alteración entre la ingesta alimentaria y el gasto calórico a consecuencia de poco movimiento o actividad física. Además de los problemas tanto orgánicos como psicológicos que causa la obesidad, esto incrementa el riesgo de sufrir hipertensión arterial, síndromes de hipoventilación y apnea obstructiva del sueño, enfermedad hepática por infiltración grasa del hígado, colelitiasis, osteoartrosis, epifisiolistesis, seudotumor cerebri, dislipidemia y, particularmente, diabetes mellitus tipo 2 .

Según documentos de la última Encuesta Nacional de Salud y Nutrición (ENSANUT-ECU 20112013), Ecuador registra un $8,6 \%$ de niños menores de 5 años con sobrepeso, mientras que, entre niños de 5 y 11 años, este índice se triplica, llegando al 29,9\%. Adicionalmente, observaciones determinan que en el Ecuador existe un crecimiento vertiginoso de la obesidad infantil, y por otro, continúa siendo alta señal de infantes con desnutrición en menores de 5 años. A pesar de que la obesidad infantil ha decaído en el país del 40,2 \% en 1986 al 25,3 \% en el 2012.

Este desequilibrio se ve intervenido por los factores de nutricionales y sedentarismo. Delimitación del problema. Los factores nutricionales es una gran clave del incremento de esta patología que afecta a los infantes en diversos niveles como socioeconómicos, ya que en muchos hogares que han migrado, dejando sus hijos con familiares o cuidadores ha causado un cambio en la dieta y alimentación hipercalórica rica grasa y azucares escasa en vitaminas, minerales y micronutrientes.

Objetivo general: Establecer orientaciones para la atención de los infantes con sobrepeso u obesidad.

Objetivos específicos: Fomentar el uso de instrumentos que permitan que accedan de manera objetiva las desviaciones no aceptables del sobrepeso de su masa o grasa corporal. Preparar el equipo de salud en la prevención y tratamiento del sobrepeso y la obesidad. Facilita acciones integradoras, en el sujeto, su familia y la comunidad, de promoción de la salud y atención primaria orientada a la comunidad.

Formulación del problema: El sedentarismo es la secuela de estilo de vida que la sociedad actual implementa que el entretenimiento de los niños esta provocando un estado que mantenga al 
cuerpo sin movimiento llevando a la falta de actividad física como el deporte o juegos que lleven que el organismo este vulnerables para el padecimiento de enfermedades cardiovascular, respiratoria, locomotor y nerviosa. Las consecuencias se presentan corto y largo plazo tenemos baja autoestima, trastornos psicoafectivos que derivan en bulimia y anorexia tornándose un círculo vicioso, enfermedades respiratorias: como apnea del sueño y asma bronquial, al riesgo cardiovascular está el aumento de la presión arterial, nivel metabólico: aumento de glucosa y lípidos en sangre.

Problemas vinculados con una mayor accesibilidad a determinados alimentos:

* aumento de la variedad de los alimentos;

* mayor disponibilidad de alimentos la comida chatarra;

*aumento de las grasas y de los hidratos de carbono en productos de bajo costo;

* menor gasto energético a causa del decrecimiento en la exigencia de las condiciones generales de vida;

*reducción de la actividad física;

* Incremento del sedentarismo;

*aumento de la cantidad de horas transcurridas frente a pantallas.

Problemas relacionados con cambios en el modelo alimenticio:

* disminución en el número de comidas familiares;

*aumento en la cantidad de comidas que los niños comen solos;

*aumento del consumo de alimentos envasados y comidas rápidas;

* caída de los patrones de consumo saludable.

\section{MATERIALES Y MÉTODOS}

La Organización Mundial de la Salud (OMS) define el sobrepeso como una desviación tipo de peso para su edad y sexo y la obesidad en edad escolar es igual a señales a su masa para su edad y sexo. Desde el punto de vista epidemiológico, la obesidad infantil se ha convertido en un problema de salud a nivel universal, para el año 2012 se estimó en 42 millones la cifra de niños de 5 años con sobrepeso. Investigaciones recientes realizadas en diferentes países manifiestan que entre el $\quad 5 \%$ y $10 \%$ de los infantes en edad escolar sufren obesidad. (OMS, 2014).

La obesidad no es un padecimiento en sí, es un factor de peligro para sufrir afecciones y disminuir la calidad de vida de los niños que la padecen. La obesidad en infantes es un asunto serio, alarmante y que nunca puede ser asumida a la ligera. (Marshall, 2012). Esto muestra el aumento sucesivo de grasa corporal no acordada a la edad de niños, adecuación a la estatura, teniendo en consideración que la magnitud en que se presenta la obesidad va aumentando con el transcurso de los años sin tomar prevención de esta. (Tansey, 2012)

La razón primordial del sobrepeso y la obesidad infantil es el desequilibrio entre el consumo y gasto calórico. El adelantamiento mundial del sobrepeso y la obesidad infantil se debe al consumo de alimentos con alto índice calórico, como grasas y azúcares, además bajos en nutrientes (vitaminas y minerales). Otra de las causas es la inactividad física, debido a la tecnología de la actualidad (videojuegos y televisión) que aumenta el sedentarismo. 
El aumento de la prevalencia de la obesidad en la 6 población pediátrica ha generado un desarrollo de enfermedades metabólicas a nivel Internacional.

Intervención nutricional En esta segunda etapa se espera una "Alimentación con gran disminución del aporte de grasa y azúcar y una mayor ingesta de fibra y agua", escogiendo alimentos de baja densidad calórica como son las frutas y los vegetales. En esencial, durante este tiempo o periodo de rápido crecimiento, las vitaminas y minerales (como el hierro o el calcio) no se vean comprometidos. La intervención dietética en el control del sobrepeso y la obesidad en niños y adolescentes ha tendido a reflejar las tendencias de gestión similares a los adultos. Las dietas bajas en grasas se han vuelto más frecuentes.

\section{RESULTADOS}

Estudios de intervención dietética en niños con sobrepeso utilizaron dietas de bajo contenido de grasa. La evidencia proporcionó resultados que muestran que la dieta no se involucra en el estado nutricional del hierro, ácido fólico, retinol y zinc. Por lo tanto, se puede finalizar que una dieta baja en grasa, en niños, es nutricionalmente segura. La mayoría de propósitos o proyectos alimenticios para el control de peso en niños sugieren la elección de alimentos bajos en grasas. Una dieta baja en grasa en general ofrece una ingesta diaria de 55 gramos, equivalentes al $25 \%$ del valor calórico total aproximadamente. Especificaciones nutricionales y dietéticas que corresponden a un segundo nivel de intervención Consumir leche, yogures y quesos, descremados. Son la mejor fuente de calcio y se necesitan porciones diarias. Seleccionar quesos de pasta blanda o untables magros. Comer una amplia variedad de carnes rojas magras y blancas, retirando la grasa visible antes de cocinar. Lo que cada uno necesita es un trozo del tamaño de la palma de la mano, una vez por día. Preferir el consumo de pescado, por lo menos una vez a la semana. Comer frutas y verduras de todo tipo y color. Cuantos más colores incluyamos, más vitaminas y minerales obtendremos. Banana, no más de una por día. Consumir una porción de vegetales en cada una de las comidas principales (almuerzo y cena). Empleando o utilizando las frutas en postres, colaciones de media mañana y exprimidas o licuadas en meriendas o desayunos. Pan, pastas, arroz y legumbres nos dan energía para nuestra actividad diaria. Dos porciones de pan blanco diarias y, tres o cuatro veces por semana, pastas, polenta, arroz o legumbres suele ser suficiente. Preparar las comidas con aceite preferentemente crudo y evitar la grasa para cocinar. Dos cucharadas soperas por día, variando entre aceite de oliva, de girasol, de maíz y de soja, son adecuadas para mantener el organismo en condiciones saludables. Evitar el uso de aderezos grasos como cremas, salsas y mayonesa. Realizar comidas diarias. Centrándose en los hábitos de consumo de grasas, es necesaria la educación alimentaria nutricional para la realización de las compras y selección de alimentos, en lugar de restringir su consumo. Los padres desempeñan un papel fundamental en las elecciones que realizan respecto a la disponibilidad de alimentos en el hogar y la preparación de estos. También es importante trabajar en la lectura de las etiquetas. Tratar de disminuir el consumo de: Lácteos con alto contenido de grasa: queso fundido, quesos duros, queso de rallar, manteca, margarina, crema. Cordero, vísceras (lengua, riñón, mondongo, corazón, molleja, achuras), salchichas y hamburguesas. Fiambres, patés, embutidos. Frituras, empanadas, pizza. Las empanadas se pueden hacer con masa dietética, rellenos con poca grasa y cocinadas al horno. Contarlas como una porción de pastas y cereales. Luego, cocinarlas al horno o sartén, 
con el mínimo de aceite. Facturas, tortas, masas, galletitas dulces rellenos. Preferir tostaditas de pan francés con dulce light, o vainillas o galletitas sin pastas de relleno. Mayonesa, sopas crema, manteca. Se pueden consumir, siempre con moderación, mayonesa y sopas-crema light. Golosinas, helados, productos de copetín. Se puede consumir una golosina pequeña por día en forma de bocadito, evitando que sea consumido en la cena. Gaseosas comunes, jugos azucarados, gelatinas comunes. Reemplazarlos por las versiones dietéticas o jugos frescos sin azúcar. Optar por agua como bebida durante las comidas. Azúcar y sal. Utilizar edulcorantes (preferentemente, sucralosa o stevia) y sal, con mucha moderación.

Denominamos barreras a los factores que pueden interferir en una adecuada evolución clínica o en el sostenimiento del niño o la familia en el cambio de estilo de vida. Si bien existe una amplia gama de factores que podrían interferir en la buena evolución del tratamiento, la mayor parte de ellos se relacionan con la falta de adhesión por parte del niño y la familia. En general, pueden ser descubiertos durante la consulta médica, pero solamente aparecerán si se indaga sobre ellos específicamente. En los niños de menor edad, las familias pueden manifestar saturación respecto de las indicaciones, mientras que en los niños mayores o adolescentes puede aparecer resistencia de parte de los propios pacientes. Algunos aspectos para considerar: Disociación entre la realidad esperada y la expectativa familiar.

Dificultad del niño o la familia para enfrentar el incumplimiento de las indicaciones, ante la figura "hegemónica" del médico. Familias desintegradas o monoparentales que no acuerdan en conjunto sobre los cambios a establecer. Distancia geográfica entre el barrio donde reside la familia y el Centro de Salud donde asiste al tratamiento. Situación laboral o económica de los padres. Falta de modelos parentales positivos

\section{Tratamiento del niño}

La mayoría de los niños con sobrepeso u obesidad son tratados en el Primer Nivel de Atención por profesionales de la salud, como pediatras, médicos de cabecera, nutricionistas, enfermeras, o bien son detectados en el transcurso de controles de salud escolar. Allí radica la importancia del entrenamiento de los mencionados agentes de salud para el primer abordaje de los niños con sobrepeso. Algunas condiciones clave deben darse en la consulta para que ésta tenga mayores probabilidades de éxito: Entre los profesionales de la salud que aborden el problema debe estar incluido un médico. Al menos dos de las siguientes intervenciones deben ser abordadas en la consulta. Intervención nutricional para controlar la ingesta. Incremento de la actividad física. Disminución de actividades sedentarias. El equipo de salud debe estar entrenado en el tema. Los padres deben estar comprometidos con el tratamiento. Se debe considerar que la mayoría de los niños con sobrepeso u obesidad no tienen problemas médicos subyacentes que causan la obesidad. Sin embargo, un porcentaje significativo podría sufrir comorbilidades relacionadas con el aumento de la adiposidad. El exceso de peso afecta negativamente la salud física y psico-social del niño y pone en peligro su salud futura. A diferencia de otros problemas de salud, la obesidad infantil tiene múltiples causas y requiere un enfoque amplio que abarque el tratamiento del paciente, la familia y el medio ambiente 
donde se desarrolla. Un enfoque motivacional, junto con cambios simples en el estilo de vida, pueden mejorar el bienestar del niño con sobrepeso y puede beneficiar potencialmente a otros miembros de la familia.

\section{CONCLUSIONES}

Los factores de riesgo pasan inadvertidos para los padres de familia, ya que no se les da la significancia que amerita, para prevenir las consecuencias que puede causar la obesidad.

Los factores de riesgo más relacionados con la obesidad en los niños estudiados fueron la falta de actividad física, el consumo de bebidas azucaradas y de alimentos fritos.

Continuar efectuando estudios que caractericen a la población estudiantil, para constituir estándares para evaluación nutricional y estructura corporal.

Realizar estudios que promuevan estilos de vida saludable y patrones de una adecuada nutrición en los niños.

\section{REFERENCIAS}

1. Bueno G, Bueno O, Moreno LA, García R, Tresaco B, Garagorri JM, Bueno M. Diversity of metabolic syndro- me risk factors in obese children and adolescents. J Physiol Biochem 2006; 62: 125-134.

2. Cole TJ, Bellizzi MC, Flegal KM, Dietz WH. Establis- hing a standard definition for child overweight and obe- sity worldwide: international survey. BMJ 2000; 320: 1240-1243.

3. Doak CM. Visscher TLS, Renders CM, Seidell JC. The prevention of overweight and obesity in children and adolescents: a review of interventions and program- mes. Obes Rev 2006; 7: 111136.

4. Moreno LA, Mesana MI, Fleta J, Ruiz JR, González- Gross MM, Sarría A, Marcos A, Bueno M and the AVE- NA Study Group. Overweight, obesity and body fat composition in Spanish adolescents. The AVENA Study. Ann Nutr Metab 2005; 49: 71-76.

5. Moreno LA, Ochoa MC, Wärnberg J, Martí M, Martí- nez JA, Marcos A. Treatment of obesity in children and adolescents: How nutrition can work? Int J Pediatr Obes 2008; 3: 72-77.

6. Moreno LA, Rodríguez G. Dietary risk factors for deve- lopment of childhood obesity. Curr Op Clin Nutr Metab Care 2007; 10: 336-341.

7. Moreno LA. Interventions to improve cardiovascular risk factors in obese children. J Pediatr Gastroenterol Nutr 2006; 43: 433-435.

8. Oude Luttikhuis $H$, Baur L, Jansen H, Shrewsbury VA, O'Malley C, Stolk RP, Summerbell CD. Interventions for treating obesity in children. Cochrane Database Syst Rev 2009; 1: CD001872.

9. Rey-López JP, Vicente-Rodríguez G, Biosca M, More- no LA. Sedentary behaviour and obesity development in children and adolescents. Nutr Metab Cardiovasc Dis 2008; 18: 242-251.

10. Summerbell CD, Waters $E$, Edmunds $L D$, et al. Inter- ventions for preventing obesity in children. Cochrane Database Syst Rev 2005; 3: CD001871. 